\title{
Association between Sour Taste SNP KCNJ2-rs236514, Diet Quality and Mild Cognitive Impairment in an Elderly Cohort
}

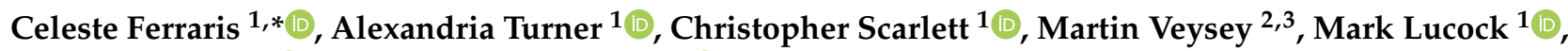 \\ Tamara Bucher 1,4 iD and Emma L. Beckett 1,4,5
}

1 School of Environmental and Life Sciences, The University of Newcastle, Ourimbah, NSW 2258, Australia; alexandria.turner@uon.edu.au (A.T.); c.scarlett@newcastle.edu.au (C.S.); mark.lucock@newcastle.edu.au (M.L.); tamara.bucher@newcastle.edu.au (T.B.); emma.beckett@newcastle.edu.au (E.L.B.)

2 School of Medicine \& Public Health, The University of Newcastle, Gosford, NSW 2250, Australia; martin.veysey@newcastle.edu.au

3 Hull York Medical School, University of Hull, Cottingham HU6 7RX, UK

4 Priority Research Centre for Physical Activity and Nutrition, The University of Newcastle, Callaghan, NSW 2308, Australia

5 Hunter Medical Research Institute, Callaghan, NSW 2308, Australia

* Correspondence: celeste.ferraris@uon.edu.au

check for updates

Citation: Ferraris, C.; Turner, A.; Scarlett, C.; Veysey, M.; Lucock, M.; Bucher, T.; Beckett, E.L. Association between Sour Taste SNP KCNJ2-rs236514, Diet Quality and Mild Cognitive Impairment in an Elderly Cohort. Nutrients 2021, 13, 719. https://doi.org/10.3390/ nu13030719

Academic Editor: Arianna Vignini

Received: 8 February 2021

Accepted: 17 February 2021

Published: 24 February 2021

Publisher's Note: MDPI stays neutral with regard to jurisdictional claims in published maps and institutional affiliations.

Copyright: (c) 2021 by the authors. Licensee MDPI, Basel, Switzerland. This article is an open access article distributed under the terms and conditions of the Creative Commons Attribution (CC BY) license (https:/ / creativecommons.org/licenses/by/ $4.0 /)$.

\begin{abstract}
Differences in sour-taste thresholds have been identified in cognition-related diseases. Diet is a modulator of cognitive health, and taste perception influences dietary preferences and habits. Heritable genetics and polymorphisms in the KCNJ2 gene involved in the transduction of sour taste have been linked to variations in sour taste and non-gustatory functions. However, relationships between sour taste genetics, mild cognitive impairment, and diet quality are yet to be elucidated. This study investigated the associations between the presence of the KCNJ2-rs236514 variant (A) allele, diet quality indices, and mild cognitive impairment evaluated by the Mini-Mental State Examination (MMSE), in a secondary cross-sectional analysis of data from the Retirement Health \& Lifestyle Study. Data from 524 elderly Australians ( $\geq 65 y$ ) were analyzed, using standard least squares regression and nominal logistic regression modeling, with demographic adjustments applied. Results showed that the presence of the KCNJ2-A allele is associated with increased proportions of participants scoring in the range indicative of mild or more severe cognitive impairment (MMSE score of $\leq 26)$ in the total cohort, and males. These associations remained statistically significant after adjusting for age, sex, and diet quality indices. The absence of association between the KCNJ2-A allele and cognitive impairment in women may be related to their higher diet quality scores in all indices. The potential link between sour taste genotype and cognitive impairment scores may be due to both oral and extra-oral functions of sour taste receptors. Further studies are required on the role and relationship of neurotransmitters, sour taste genotypes and sour taste receptors in the brain, and dietary implications, to identify potential risk groups or avenues for therapeutic or prophylactic interventions.
\end{abstract}

Keywords: sour; taste; genetics; KCNJ2 gene; rs236514; polymorphism; SNP; cognitive impairment; dementia; elderly; diet

\section{Introduction}

As the population ages, cognition-related disease prevalence in Australia and the directly associated costs are expected to rise by $90 \%$ over the next twenty years [1]. Worldwide, a 300\% increase in dementia prevalence is expected by 2050 [2]. The significant impact on quality of life [3,4] and the relationship to increased risk of concurrent chronic diseases [5] highlights an immediate need for novel management strategies and broader understandings of the risk factors associated with the onset of cognitive decline.

Diet is a known modulator of cognitive health, [6-8] and is established as a preventative tool in cognition-related diseases [6,9]. Dietary preferences are strongly influenced by 
perception of the five key tastes - bitter, sweet, sour, salt, and umami $[10,11]$. Sour taste is stimulated by acids in foods [12] such as berries, citrus fruits, and fermented foods [13]. These sour foods are commonly consumed in diets found to be effective in the prevention of cognitive decline [6-8]. The relationships between differences in taste perception and intake of cognitive-health-promoting foods is an under-researched area, particularly for sour taste.

Recent research has demonstrated loss of taste function in mild cognitive impairment (MCI), dementia [14,15], and Alzheimer's disease (AD) [15-17]. Specifically, for sour taste, sour-threshold testing has revealed that higher concentrations are required before sourness is detected by patients with dementia and $\mathrm{AD}[14,16]$. Taste decline in cognitive impairment has been found to be independent of other influencing factors, such as prescription drugs, salivation, zinc levels [15], and ageing [17,18]. However, the direction and consequences of the associations have yet to be established. Therefore, the more direct relationships between sour taste and cognition require further investigation.

Sour-taste thresholds and perception are modulated by receptor genetics. Early research has shown heritable genetics significantly contribute to variations in sour recognition thresholds [19], perceived pleasantness and intensity of sour taste, and the frequency with which sour foods are consumed [20]. Single-nucleotide polymorphisms (SNPs) in taste receptors have been associated with alterations in taste, including sour perception [10]. Taste-receptor SNPs that increase intensity of taste perception have been shown to reduce liking of tastants [21,22]. The KCNJ2 (Potassium Inwardly Rectifying Channel Subfamily J Member 2) gene is found in the Type III sour-sensing taste cells [23] and is linked to the magnitude of sour-taste transduction [24]. One study found that carriers of the KCNJ2rs236514 variant allele (A) had a higher preference (liking) for sour [25], which may suggest that the SNP reduces transduction. Whether the SNP and reduced sensitivity to sour are related to risk for cognition-related diseases has yet to be elucidated.

Therefore, the possible relationship between KCNJ2-rs236514 sour genotype, diet quality, and a marker of cognitive impairment was assessed in a well-characterized elderly cohort. This cross-sectional analysis used scores from the Mini-Mental State Examination (MMSE) as an index for cognitive impairment and assessed the relationship between this index and the common sour-taste SNP, KCNJ2-rs236514, and three diet-quality indices.

\section{Materials and Methods}

\subsection{Subjects}

Data for this secondary analysis were obtained from a cross-sectional cohort of elderly subjects ( $\geq 65$ years) from the Retirement Health and Lifestyle Study (RHLS) [26-28]. Randomly selected participants lived independently or in retirement villages in the local Gosford and Wyong state government areas of the Central Coast region of NSW, Australia. There were no exclusion criteria based on pre-existing health conditions, cognitive or otherwise; however, participants were required to have sufficient language and cognition skills to be able to provide written informed consent. From the 831 participants that took part in the initial study, only those who provided blood samples and valid food-frequency questionnaires (FFQ) were genotyped for KCNJ2-rs236514 and were hence included in the current study $(n=524)$. The Human Research Ethics Committee of the University of Newcastle granted ethics approval (Reference No. H-2008-0431) [26-28].

\subsection{Demographics and Anthropometrics}

Interviewer-administered questionnaires collected data on age, sex, education, income, and smoking history [26-28]. Body Mass Index $\left(B M I=\right.$ weight $(\mathrm{kg}) /$ height $\left.\left(\mathrm{m}^{2}\right)\right)$ was calculated by using height and weight measures obtained by following the International Society for the Advancement of Kinanthropometry (ISAK) guidelines [29]. Height was recorded to the nearest $0.01 \mathrm{~cm}$, using the stretch stature method. Weight was recorded to the nearest $0.01 \mathrm{~kg}$ on digital scales (Wedderburn(C) UWPM150 Platform Scale). 


\subsection{Genotyping}

Whole fasted blood samples were collected into EDTA-lined, tubes and DNA were isolated from peripheral blood cells $[28,30]$ following the manufacturer's instructions (QIAGEN QIAmp DNA mini-kit) [31]. Both the blood samples and DNA were stored at $-20{ }^{\circ} \mathrm{C}[28,30]$. TaqMan assay (Applied Biosystems, ThermoFisher Scientific, Waltham, MA, USA 02451) was used to genotype the KCNJ2-rs236514 allelic variants, according to the manufacturer's protocols.

\subsection{Cognitive Assessment}

Cognitive function was assessed with the MMSE, a valid and reliable tool widely used and recommended in research [32,33]. The MMSE measures orientation, registration, attention, calculation, memory, recall, and drawing ability to diagnose dementia [34]. The threshold of $\leq 26$ was used to classify those with $\mathrm{MCI}$, inclusive of those with more severe forms of cognitive impairment. [35-39].

\subsection{Diet Quality Indices}

Dietary data were obtained by a previously validated FFQ [40]. Foodworks ${ }^{\mathrm{TM}}$ (V.2.10.146) software was used to analyze participants' intake of the 225 food items [41]. An FFQ was deemed invalid if data were incomplete or if energy intake reports were $>30,000 \mathrm{~kJ} / \mathrm{d}$ or $<3000 \mathrm{~kJ} / \mathrm{d}$.

Three diet-quality indices were generated from the FFQ data. The Dietary Guideline Index (DGI) is a 150-point index based on Australian dietary and alcohol intake guidelines [42,43] and national indicators of food and nutrition [44]. The DGI provides a measure of diet quality that reflects dietary diversity, key nutrient intake from core food groups, and healthful food (e.g., vegetables) and unhealthy food intakes [26,45]. The Australian Recommended Food Score (ARFS) is a 74-point index based on the Australian Dietary Guidelines that focuses on variety as the key indicator of diet quality [26,46]. Finally, the Australian Healthy Eating Index (Aust-HEI) is a 60-point index that considers variety, adherence to healthy food-choice guidelines; higher consumption of fruits, vegetables, and low-fat milk; and lower consumption of meat, saturated fats, and low-nutrient-density foods to be a diet of higher quality $[26,47]$.

\subsection{Statistical Analysis}

Data were analyzed, using JMP (Pro V.14.2.0; SAS Institute Inc., Cary, NC, USA 27513). Continuous variable distributions (means, 95\% confidence intervals and standard deviations) and categorical variable distributions (number and percentage of cohort) describe the cohort characteristics. Where categories had insufficient numbers of participants for statistical analysis, groups were consolidated for further analyses. Ex-smokers and current smokers were collapsed into a single "history of smoking" category; income was collapsed into two categories $(\leq \$ 20,000$ and $>$ AUD $\$ 20,000$ per year), and education categories were $\leq$ Trade qualification and TAFE (Technical and Further Education) or higher. As previous research has demonstrated a potential sex dimorphism in sour-taste perception, with women finding sour more intense and having higher sensitivity to sourness [48-50], analyses were stratified by sex.

KCNJ2-rs236154 allele frequency was reported as number and percentage of the study cohort and analyzed by presence or absence of the KCNJ2 variant allele (A). MMSE scores were categorized nominally based on the defined thresholds $(\leq 26=\mathrm{MCI} ;>26=$ normal cognition). Statistical significance of continuous variables was examined through standard least squares regression and for categorical variables through nominal logistic regression $\left(\chi^{2}, p\right.$-values, odds ratios, and $95 \%$ confidence intervals). Analyses were adjusted for age, sex, education, income, smoking status, and BMI. As diet is a contributing factor to cognitive health $[6,7]$, and diet is modified by taste perception, further analyses with adjustments for the diet quality indices were made. The $p$-values are presented to the first significant figure, and threshold $p$-values of $<0.05$ were considered statistically significant. 


\section{Results}

\subsection{Participant Characteristics}

The ages of the 524 participants ranged from 65 to 94 years (mean 77.6 years, $S D \pm 6.7$ ) (Table 1). The mean BMI was $28.6 \mathrm{~kg} / \mathrm{m}^{2}$ (Table 1). Age and BMI did not vary by sex. The mean diet-quality scores were $96.8 / 150$ points (DGI), $29.0 / 74$ points (ARFS), and $30.3 / 60$ points (Aust-HEI) (Table 1). Females had higher mean diet quality scores than males across all three diet quality indices (DGI: $p=0.0005$, ARFS: $p=0.003$ and Aust-HEI: $p=<0.0001$, Table 1 ).

Table 1. Distribution of continuous variables by total cohort and by sex.

\begin{tabular}{|c|c|c|c|c|c|c|c|c|c|c|}
\hline \multirow[b]{2}{*}{ Variable } & \multicolumn{3}{|c|}{ Total } & \multicolumn{3}{|c|}{ Females } & \multicolumn{3}{|c|}{ Males } & \multirow[b]{2}{*}{$p$} \\
\hline & $\begin{array}{l}\text { Mean } \\
\text { (SD) }\end{array}$ & Min & $\operatorname{Max}$ & $\begin{array}{l}\text { Mean } \\
\text { (SD) }\end{array}$ & Min & $\operatorname{Max}$ & $\begin{array}{l}\text { Mean } \\
\text { (SD) }\end{array}$ & Min & Max & \\
\hline $\begin{array}{c}\text { Age } \\
\text { (years) }\end{array}$ & $77.6( \pm 6.7)$ & 65.0 & 94.0 & $\begin{array}{c}77.7 \\
( \pm 6.7)\end{array}$ & 65 & 94 & $\begin{array}{c}77.4 \\
( \pm 6.8)\end{array}$ & 65 & 93 & 0.6 \\
\hline $\begin{array}{c}\text { BMI } \\
\left(\mathrm{kg} / \mathrm{m}^{2}\right)\end{array}$ & $28.6( \pm 4.8)$ & 17.1 & 46.3 & $\begin{array}{c}28.6 \\
( \pm 5.0)\end{array}$ & 17.6 & 46.3 & $\begin{array}{c}28.6 \\
( \pm 4.5)\end{array}$ & 17.1 & 45.4 & 0.9 \\
\hline DGI & $\begin{array}{c}96.8 \\
( \pm 15.9)\end{array}$ & 30.9 & 132.6 & $\begin{array}{c}99.0 \\
( \pm 16.3)\end{array}$ & 30.9 & 132.6 & $\begin{array}{c}94.2 \\
( \pm 15.0)\end{array}$ & 51.8 & 130.4 & 0.0005 \\
\hline ARFS & $29.0( \pm 8.0)$ & 6.0 & 50.0 & $\begin{array}{c}29.9 \\
( \pm 8.1)\end{array}$ & 6 & 50 & $\begin{array}{c}27.8 \\
( \pm 7.7)\end{array}$ & 10 & 49 & 0.003 \\
\hline Aust-HEI & $30.3( \pm 9.5)$ & 4.9 & 50.8 & $\begin{array}{c}32.0 \\
( \pm 9.1)\end{array}$ & 6.4 & 50.8 & $\begin{array}{c}28.3 \\
( \pm 9.7)\end{array}$ & 4.9 & 46.5 & $<0.0001$ \\
\hline
\end{tabular}

SD, standard deviation; BMI, Body Mass Index; DGI, Dietary Guideline Index (150 points); ARFS, Australian Recommended Food Score (74 points); Aust-HEI, Australian Health Eating Index (60 points).

The cohort was $54.4 \%$ female (Table 2). Most participants reported earning > AUD $\$ 20,000$ per year and being educated at TAFE level or higher (Table 2). Men were more likely than women to have higher incomes ( $85.6 \%$ vs. $53.8 \%, p=<0.0001)$, to be educated at TAFE level or higher $(75.7 \%$ vs. $60.2 \%, p=0.0001)$, and to have a history of smoking $(66.5 \%$ vs. $35.1 \%, p=<0.0001$ ) (Table 2).

Table 2. Distribution of categorical variables by total cohort and by sex.

\begin{tabular}{|c|c|c|c|c|}
\hline \multirow{2}{*}{ Variable } & Total & Females & Males & \multirow{2}{*}{$p$} \\
\hline & $n(\%)$ & $n(\%)$ & $n(\%)$ & \\
\hline \multicolumn{5}{|l|}{ Sex } \\
\hline Males & $239(45.6)$ & & & \\
\hline Females & $285(54.4)$ & & & \\
\hline \multicolumn{5}{|l|}{ Income } \\
\hline$\leq$ AUD $\$ 20,000$ per year & $161(31.5)$ & $127(46.2)$ & $34(14.4)$ & $<0.0001$ \\
\hline$>$ AUD $\$ 20,000$ per year & $350(68.5)$ & $148(53.8)$ & $202(85.6)$ & \\
\hline \multicolumn{5}{|l|}{ Education } \\
\hline$\leq$ Trade qualification & $171(32.7)$ & $113(39.8)$ & $58(24.3)$ & 0.0001 \\
\hline TAFE or higher & $352(67.3)$ & $171(60.2)$ & $181(75.7)$ & \\
\hline \multicolumn{5}{|l|}{ Smoking } \\
\hline History of smoking & $259(49.4)$ & $100(35.1)$ & $159(66.5)$ & $<0.0001$ \\
\hline Never smoked & $265(50.6)$ & $185(64.9)$ & $80(33.5)$ & \\
\hline
\end{tabular}

TAFE, Technical and Further Education.

\subsection{Genotype Distributions}

The frequency of the KCNJ2-rs236514 variant allele (A) was 0.56 , and the ancestral allele $(\mathrm{G})$ was 0.44 . A large proportion of the participants (81.1\%) carried the KCNJ2-A allele (AA or AG genotypes), and there were no differences by sex (Table 3 ). 
Table 3. KCNJ2-rs236514 variant (A) allele distributions by total cohort and by sex.

\begin{tabular}{ccccc}
\hline \multirow{2}{*}{ Genotype } & Total & Females & Males & \multirow{p}{*}{} \\
\cline { 2 - 4 } & $\boldsymbol{n ( \% )}$ & $\boldsymbol{n ( \% )}$ & $\boldsymbol{n ( \% )}$ & \\
\hline KCNJ2-A allele present & $425(81.1)$ & $236(82.8)$ & $189(79.0)$ & \\
KCNJ2-A allele absent & $99(18.9)$ & $49(17.2)$ & $50(20.9)$ & 0.3 \\
\hline
\end{tabular}

\subsection{MMSE Distributions}

MMSE scores indicative of MCI occurred in 17.6\% of the cohort, $16.5 \%$ of females, and $18.8 \%$ of males (Table 4 ). There were no statistically significant differences by sex.

Table 4. MMSE distributions by total cohort and by sex.

\begin{tabular}{|c|c|c|c|c|}
\hline \multirow{2}{*}{ MMSE } & Total & Female & Male & \multirow{2}{*}{$p$} \\
\hline & $n(\%)$ & $n(\%)$ & $n(\%)$ & \\
\hline $\mathrm{MCI}(\leq 26)$ & $92(17.6)$ & $47(16.5)$ & $45(18.8)$ & \multirow{2}{*}{0.5} \\
\hline Normal cognition (>27) & $432(82.4)$ & $238(83.5)$ & $194(81.2)$ & \\
\hline
\end{tabular}

MMSE, Mini-Mental State Examination; MCI, mild cognitive impairment.

\subsection{Relationships between Presence of the KCNJ2-rs236514 Variant (A) Allele and Confounding Variables}

The presence of the KCNJ2-rs236514 variant (A) allele was more likely in older females $(p=0.04)$ and was associated with higher BMI in males $(p=0.002)$ (Table 5). There were no differences in the presence of the KCNJ2-A allele in the distributions of sex, income, education, and smoking ( $x^{2}$ range $=0.7-2.5$, all $p$-values $\geq 0.05$ ).

Table 5. Demographic and clinical characteristics by presence of the KCNJ2-rs236514 variant (A) allele in the total cohort and by sex (continuous variables).

\begin{tabular}{|c|c|c|c|c|c|c|c|c|c|}
\hline \multirow{3}{*}{ Variable } & \multicolumn{3}{|c|}{ Total } & \multicolumn{3}{|c|}{ Females } & \multicolumn{3}{|c|}{ Males } \\
\hline & \multicolumn{2}{|c|}{$\begin{array}{c}\text { LSM } \\
(95 \% \text { CoI })\end{array}$} & \multirow{2}{*}{$p$} & \multicolumn{2}{|c|}{$\begin{array}{c}\text { LSM } \\
(95 \% \text { CoI) }\end{array}$} & \multirow{2}{*}{$p$} & \multicolumn{2}{|c|}{$\begin{array}{c}\text { LSM } \\
(95 \% \text { CoI })\end{array}$} & \multirow{2}{*}{$p$} \\
\hline & $\begin{array}{c}\text { A Allele } \\
\text { Present }\end{array}$ & $\begin{array}{c}\text { A Allele } \\
\text { Absent }\end{array}$ & & $\begin{array}{l}\text { A Allele } \\
\text { Present }\end{array}$ & $\begin{array}{c}\text { A Allele } \\
\text { Absent }\end{array}$ & & $\begin{array}{c}\text { A Allele } \\
\text { Present }\end{array}$ & $\begin{array}{c}\text { A Allele } \\
\text { Absent }\end{array}$ & \\
\hline $\begin{array}{c}\text { Age } \\
\text { (years) }\end{array}$ & $\begin{array}{c}77.8 \\
(77.2-78.5)\end{array}$ & $\begin{array}{c}76.5 \\
(75.1-77.8)\end{array}$ & 0.07 & $\begin{array}{c}78.1 \\
(77.2-78.9)\end{array}$ & $\begin{array}{c}75.9 \\
(73.2-78.9)\end{array}$ & 0.04 & $\begin{array}{c}77.5 \\
(76.5-78.5)\end{array}$ & $\begin{array}{c}77.0 \\
(75.1-78.9)\end{array}$ & 0.7 \\
\hline $\begin{array}{c}\mathrm{BMI} \\
\left(\mathrm{kg} / \mathrm{m}^{2}\right)\end{array}$ & $\begin{array}{c}28.7 \\
(28.2-29.2)\end{array}$ & $\begin{array}{c}28.0 \\
(27.0-28.9)\end{array}$ & 0.2 & $\begin{array}{c}28.4 \\
(27.7-29.1)\end{array}$ & $\begin{array}{c}29.1 \\
(27.6-30.5)\end{array}$ & 0.4 & $\begin{array}{c}29.0 \\
(28.4-28.1)\end{array}$ & $\begin{array}{c}26.8 \\
(25.6-28.1)\end{array}$ & 0.002 \\
\hline DGI & $\begin{array}{c}97.2 \\
(95.7-98.7)\end{array}$ & $\begin{array}{c}94.9 \\
(91.8-98.0)\end{array}$ & 0.2 & $\begin{array}{c}99.7 \\
(93.1-102.3)\end{array}$ & $\begin{array}{c}97.7 \\
(93.2-101.3)\end{array}$ & 0.5 & $\begin{array}{c}94.7 \\
(92.5-96.8)\end{array}$ & $\begin{array}{c}92.2 \\
(88.0-96.4)\end{array}$ & 0.3 \\
\hline ARFS & $\begin{array}{c}28.9 \\
(28.1-29.7)\end{array}$ & $\begin{array}{c}29.1 \\
(27.5-30.7)\end{array}$ & 0.9 & $\begin{array}{c}29.5 \\
(28.5-30.5)\end{array}$ & $\begin{array}{c}31.9 \\
(29.7-34.2)\end{array}$ & 0.06 & $\begin{array}{c}28.2 \\
(24.2-28.4)\end{array}$ & $\begin{array}{c}26.3 \\
(24.2-28.4)\end{array}$ & 0.1 \\
\hline Aust-HEI & $\begin{array}{c}30.5 \\
(29.6-31.4)\end{array}$ & $\begin{array}{c}29.5 \\
(27.7-31.4)\end{array}$ & 0.4 & $\begin{array}{c}32.0 \\
(30.8-33.2)\end{array}$ & $\begin{array}{c}32.2 \\
(29.6-34.7)\end{array}$ & 0.9 & $\begin{array}{c}28.7 \\
(27.3-30.0)\end{array}$ & $\begin{array}{c}27.0 \\
(24.3-29.7)\end{array}$ & 0.3 \\
\hline
\end{tabular}

LSM, least squares mean; CoI, confidence interval; BMI, Body Mass Index; DGI, Dietary Guideline Index (150 points); ARFS, Australian Recommended Food Score (74 points); Aust-HEI, Australian Health Eating Index (60 points).

\subsection{Relationships between MMSE Scores and Confounding Variables}

In the total cohort and males, MMSE scores indicative of MCI were associated with lower diet-quality scores (ARFS) ( $p=0.002, p=0.0004$ respectively) (Table 6). Older women were more likely to have MMSE scores indicative of MCI than younger women $(p=0.009)$ (Table 6). Lower education was associated with an increased likelihood of meeting the MMSE threshold score indicative of MCI in the total cohort, and in women $(p=0.04$, $p=0.02$ respectively) (Table 7 ). 
Table 6. Demographic and clinical characteristics by MMSE scores indicative of MCI in the total cohort and by sex (continuous variables).

\begin{tabular}{|c|c|c|c|c|c|c|c|c|c|}
\hline \multirow{3}{*}{ Variable } & \multicolumn{3}{|c|}{ Total } & \multicolumn{3}{|c|}{ Females } & \multicolumn{3}{|c|}{ Males } \\
\hline & \multicolumn{2}{|c|}{$\begin{array}{c}\text { MMSE } \\
\text { LSM } \\
(95 \% \text { CoI })\end{array}$} & \multirow[t]{2}{*}{$p$} & \multicolumn{2}{|c|}{$\begin{array}{c}\text { MMSE } \\
\text { LSM } \\
(95 \% \text { CoI })\end{array}$} & $p$ & \multicolumn{2}{|c|}{$\begin{array}{c}\text { MMSE } \\
\text { LSM } \\
(95 \% \text { CoI) }\end{array}$} & \multirow[t]{2}{*}{$p$} \\
\hline & $\leq \mathbf{2 6}$ & $>27$ & & $\leq \mathbf{2 6}$ & $>27$ & & $\leq \mathbf{2 6}$ & $>27$ & \\
\hline $\begin{array}{c}\text { Age } \\
\text { (years) }\end{array}$ & $\begin{array}{c}78.4 \\
(77.0-79.8)\end{array}$ & $\begin{array}{c}77.4 \\
(76.7-78.0)\end{array}$ & 0.2 & $\begin{array}{c}80.1 \\
(78.1-82.0)\end{array}$ & $\begin{array}{c}77.2 \\
(76.4-78.1)\end{array}$ & 0.009 & $\begin{array}{c}76.7 \\
(74.7-78.8)\end{array}$ & $\begin{array}{c}77.6 \\
(76.6-78.5)\end{array}$ & 0.5 \\
\hline $\begin{array}{c}\mathrm{BMI} \\
\left(\mathrm{kg} / \mathrm{m}^{2}\right)\end{array}$ & $\begin{array}{c}28.7 \\
(27.6-29.7)\end{array}$ & $\begin{array}{c}28.5 \\
(28.1-29.0)\end{array}$ & 0.8 & $\begin{array}{c}28.4 \\
(26.8-30.0)\end{array}$ & $\begin{array}{c}28.6 \\
(27.9-29.2)\end{array}$ & 0.8 & $\begin{array}{c}28.9 \\
(27.5-30.3)\end{array}$ & $\begin{array}{c}28.5 \\
(27.8-29.1)\end{array}$ & 0.6 \\
\hline DGI & $\begin{array}{c}94.7 \\
(91.4-98.1)\end{array}$ & $\begin{array}{c}97.2 \\
(95.7-98.7)\end{array}$ & 0.2 & $\begin{array}{c}97.3 \\
(92.5-102.1)\end{array}$ & $\begin{array}{c}99.3 \\
(97.2-101.3)\end{array}$ & 0.5 & $\begin{array}{c}92.1 \\
(87.5-96.6)\end{array}$ & $\begin{array}{c}94.6 \\
(92.5-96.7)\end{array}$ & 0.3 \\
\hline ARFS & $\begin{array}{c}26.6 \\
(24.9-28.2)\end{array}$ & $\begin{array}{c}29.4 \\
(28.7-30.2)\end{array}$ & 0.002 & $\begin{array}{c}29.0 \\
(26.6-31.4)\end{array}$ & $\begin{array}{c}30.1 \\
(29.1-31.1)\end{array}$ & 0.4 & $\begin{array}{c}24.1 \\
(21.8-26.3)\end{array}$ & $\begin{array}{c}28.6 \\
(27.6-29.7)\end{array}$ & 0.0004 \\
\hline Aust-HEI & $\begin{array}{c}29.4 \\
(27.4-31.4)\end{array}$ & $\begin{array}{c}30.5 \\
(29.6-31.4)\end{array}$ & 0.3 & $\begin{array}{c}32.6 \\
(30.0-35.2)\end{array}$ & $\begin{array}{c}31.9 \\
(30.8-33.1)\end{array}$ & 0.7 & $\begin{array}{c}26.1 \\
(23.2-29.0)\end{array}$ & $\begin{array}{c}28.8 \\
(27.4-30.2)\end{array}$ & 0.1 \\
\hline
\end{tabular}

MMSE, Mini-Mental State Examination; LSM, least squares mean; CoI, confidence interval; BMI, Body Mass Index; DGI, Dietary Guideline Index (150 points); ARFS, Australian Recommended Food Score (74 points); Aust-HEI, Australian Health Eating Index (60 points).

Table 7. Demographic characteristics by MMSE scores indicative of MCI in the total cohort and by sex (categorical variables).

\begin{tabular}{|c|c|c|c|c|c|c|c|c|c|}
\hline \multirow{3}{*}{ Variable } & \multicolumn{3}{|c|}{ Total } & \multicolumn{3}{|c|}{ Females } & \multicolumn{3}{|c|}{ Males } \\
\hline & \multicolumn{2}{|c|}{$\begin{array}{c}\text { MMSE } \\
\text { LSM } \\
(95 \% \text { CoI) }\end{array}$} & \multirow[t]{2}{*}{$p$} & \multicolumn{2}{|c|}{$\begin{array}{c}\text { MMSE } \\
\text { LSM } \\
(95 \% \text { CoI) }\end{array}$} & $p$ & \multicolumn{2}{|c|}{$\begin{array}{c}\text { MMSE } \\
\text { LSM } \\
(95 \% \text { CoI) }\end{array}$} & \multirow[t]{2}{*}{$p$} \\
\hline & $\leq 26$ & $>27$ & & $\leq 26$ & $>27$ & & $\leq 26$ & $>27$ & \\
\hline Sex & $\begin{array}{c}0.8 \\
(0.5-1.2)\end{array}$ & $\begin{array}{c}1.3 \\
(0.8-2.0)\end{array}$ & 0.3 & & & & & & \\
\hline Income & $\begin{array}{c}0.8 \\
(0.5-1.3)\end{array}$ & $\begin{array}{c}1.3 \\
(0.8-2.1)\end{array}$ & 0.3 & $\begin{array}{c}0.8 \\
(0.4-1.4)\end{array}$ & $\begin{array}{c}1.3 \\
(0.7-2.4)\end{array}$ & 0.4 & $\begin{array}{c}1.0 \\
(0.4-2.4)\end{array}$ & $\begin{array}{c}1.0 \\
(0.4-2.6)\end{array}$ & 0.9 \\
\hline Education & $\begin{array}{c}0.6 \\
(0.4-0.9)\end{array}$ & $\begin{array}{c}1.6 \\
(1.0-2.6)\end{array}$ & 0.04 & $\begin{array}{c}0.5 \\
(0.3-0.9)\end{array}$ & $\begin{array}{c}2.2 \\
(1.2-4.1)\end{array}$ & 0.02 & $\begin{array}{c}0.8 \\
(0.4-1.7)\end{array}$ & $\begin{array}{c}1.3 \\
(0.6-2.7)\end{array}$ & 0.5 \\
\hline Smoking & $\begin{array}{c}0.7 \\
(0.5-1.1)\end{array}$ & $\begin{array}{c}1.4 \\
(0.9-2.2)\end{array}$ & 0.1 & $\begin{array}{c}1.0 \\
(0.5-1.9)\end{array}$ & $\begin{array}{c}1.0 \\
(0.5-1.9)\end{array}$ & 1.0 & $\begin{array}{c}0.5 \\
(0.2-1.0)\end{array}$ & $\begin{array}{c}1.0 \\
(1.0-4.2)\end{array}$ & 0.05 \\
\hline
\end{tabular}

MMSE, Mini-Mental State Examination; LSM, least squares mean; CoI, confidence interval.

\subsection{Relationships between KCNJ2-rs236514 and MCI (MMSE)}

Those with the KCNJ2-A allele were more likely to have MMSE scores indicative of MCI in the unadjusted $(p=0.03)$ and age and sex-adjusted models $(p=0.04)$, but not in the fully adjusted model $(p=0.09)$ (Table 8$)$. After stratifying by sex, male KCNJ2-A allele carriers were more likely to meet the MMSE threshold scores for MCI than non-carriers, in the unadjusted $(p=0.02)$ and age-adjusted $(p=0.02)$ models (Table 9$)$. There were no relationships found between MCI (MMSE) and the categorical variables amongst women. 
Table 8. Odds of MMSE scores indicative of MCI by KCNJ2-A allele presence in the total cohort, in unadjusted and adjusted models.

\begin{tabular}{ccccccc}
\hline & \multicolumn{2}{c}{ Unadjusted } & \multicolumn{2}{c}{ Model 1 } & \multicolumn{2}{c}{ Model 2 } \\
\cline { 2 - 7 } & $x^{2}$ & OR & $x^{2}$ & OR & $x^{2}$ & OR \\
& $(p)$ & $(\mathbf{9 5 \%}$ CoI) & $(p)$ & $(\mathbf{9 5 \%}$ CoI) & $(p)$ & $\mathbf{( 9 5 \% ~ C o I ) ~}$ \\
\hline MMSE & 4.3 & 2.0 & 4.1 & 2.0 & 2.8 & 1.8 \\
$\leq 26$ & $(0.03)$ & $(1.0-4.0)$ & $(0.04)$ & $(1.0-4.0)$ & $(0.09)$ & $(1.0-3.7)$ \\
\hline
\end{tabular}

MMSE, Mini-Mental State Examination; OR, odds ratio; CoI, confidence interval; Model 1, adjusted for age and sex; Model 2, adjusted for age, sex, income, education, smoking, and BMI.

Table 9. Odds of MMSE scores indicative of MCI by KCNJ2-A allele presence in unadjusted and adjusted models, by sex.

\begin{tabular}{|c|c|c|c|c|c|c|c|c|c|c|c|c|}
\hline & \multicolumn{6}{|c|}{ Females } & \multicolumn{6}{|c|}{ Males } \\
\hline & \multicolumn{2}{|c|}{ Unadjusted } & \multicolumn{2}{|c|}{ Model 1} & \multicolumn{2}{|c|}{ Model 2} & \multicolumn{2}{|c|}{ Unadjusted } & \multicolumn{2}{|c|}{ Model 1} & \multicolumn{2}{|c|}{ Model 2} \\
\hline & $\begin{array}{l}x^{2} \\
(p)\end{array}$ & $\begin{array}{c}\text { OR } \\
(95 \% \text { CoI) }\end{array}$ & $\begin{array}{l}x^{2} \\
(p)\end{array}$ & $\begin{array}{c}\text { OR } \\
(95 \% \text { CoI) }\end{array}$ & $\begin{array}{l}x^{2} \\
(p)\end{array}$ & $\begin{array}{c}\text { OR } \\
(95 \% \text { CoI) }\end{array}$ & $\begin{array}{l}x^{2} \\
(p)\end{array}$ & $\begin{array}{c}\text { OR } \\
(95 \% \text { CoI) }\end{array}$ & $\begin{array}{l}x^{2} \\
(p)\end{array}$ & $\begin{array}{c}\text { OR } \\
(95 \% \text { CoI) }\end{array}$ & $\begin{array}{l}x^{2} \\
(p)\end{array}$ & $\begin{array}{c}\text { OR } \\
\text { (95\% CoI) }\end{array}$ \\
\hline$\underset{\leq 26}{\operatorname{MMSE}}$ & $\begin{array}{c}0.6 \\
(0.4)\end{array}$ & $\begin{array}{c}1.4 \\
(0.6-3.6)\end{array}$ & $\begin{array}{c}0.2 \\
(0.7)\end{array}$ & $\begin{array}{c}1.2 \\
(0.5-3.1)\end{array}$ & $\begin{array}{c}0.1 \\
(0.7)\end{array}$ & $\begin{array}{c}1.2 \\
(0.5-3.1)\end{array}$ & $\begin{array}{c}5.0 \\
(0.02)\end{array}$ & $\begin{array}{c}3.0 \\
(1.0-8.8)\end{array}$ & $\begin{array}{c}5.1 \\
(0.02)\end{array}$ & $\begin{array}{c}3.0 \\
(1.0-8.9)\end{array}$ & $\begin{array}{c}3.4 \\
(0.06)\end{array}$ & $\begin{array}{c}2.7 \\
(0.9-8.2)\end{array}$ \\
\hline
\end{tabular}

MMSE, Mini-Mental State Examination; OR, odds ratio; CoI, confidence interval; Model 1, adjusted for age; Model 2, adjusted for age, income, education, smoking, and BMI.

\subsection{Relationships between KCNJ2-rs236514 and MMSE Scores Indicative of MCI, Adjusting for} the Diet Quality Indices

As scores on the ARFS index were associated with MCI (MMSE) (Table 6), further analyses were conducted adjusting for the diet-quality indices (Tables 10 and 11). The relationship between the presence of the KCNJ2-A allele and MMSE scores indicative of MCI remained significant after adjusting for each of the diet-quality indices in the total cohort (DGI: $p=0.04$; ARFS: $p=0.03$; and Aust-HEI: $p=0.03$; Table 10), and in men (DGI: $p=0.02$; ARFS: $p=0.007$; and Aust-HEI: $p=0.02$; Table 11).

Table 10. Odds of MMSE scores indicative of MCI by KCNJ2-A allele presence in the total cohort, in models adjusting for diet quality.

\begin{tabular}{ccccccc}
\hline & \multicolumn{2}{c}{ Model 1 } & \multicolumn{2}{c}{ Model 2 } & \multicolumn{2}{c}{ Model 3 } \\
\cline { 2 - 7 } & $x^{\mathbf{2}}$ & OR & $x^{\mathbf{2}}$ & OR & $x^{\mathbf{2}}$ & OR \\
& $(p)$ & $(\mathbf{9 5 \%}$ CoI) & $(p)$ & $(\mathbf{9 5 \%}$ CoI) & $(p)$ & $\mathbf{( 9 5 \% ~ C o I ) ~}$ \\
\hline MMSE & 4.3 & 2.0 & 4.5 & 2.0 & 4.5 & 2.0 \\
$\leq 26$ & $(0.04)$ & $(1.0-4.0)$ & $(0.03)$ & $(1.0-4.1)$ & $(0.03)$ & $(1.0-4.1)$ \\
\hline
\end{tabular}

MMSE, Mini-Mental State Examination; OR, odds ratio; CoI, confidence interval; Model 1, adjusted for DGI (Dietary Guideline Index); Model 2, adjusted for ARFS (Australian Recommended Food Score); Model 3, adjusted for Aust-HEI (Australian Health Eating Index).

Table 11. Odds of MMSE scores indicative of MCI by KCNJ2-A allele presence in models adjusting for diet quality, by sex.

\begin{tabular}{|c|c|c|c|c|c|c|c|c|c|c|c|c|}
\hline & \multicolumn{6}{|c|}{ Females } & \multicolumn{6}{|c|}{ Males } \\
\hline & \multicolumn{2}{|c|}{ Model 1} & \multicolumn{2}{|c|}{ Model 2} & \multicolumn{2}{|c|}{ Model 3} & \multicolumn{2}{|c|}{ Model 1} & \multicolumn{2}{|c|}{ Model 2} & \multicolumn{2}{|c|}{ Model 3} \\
\hline & $\begin{array}{l}x^{2} \\
(p)\end{array}$ & $\begin{array}{c}\text { OR } \\
(95 \% \text { CoI })\end{array}$ & $\begin{array}{l}x^{2} \\
(p)\end{array}$ & $\begin{array}{c}\text { OR } \\
(95 \% \text { CoI })\end{array}$ & $\begin{array}{l}x^{2} \\
(p)\end{array}$ & $\begin{array}{c}\text { OR } \\
(95 \% \text { CoI })\end{array}$ & $\begin{array}{l}x^{2} \\
(p)\end{array}$ & $\begin{array}{c}\text { OR } \\
(95 \% \text { CoI })\end{array}$ & $\begin{array}{l}x^{2} \\
(p)\end{array}$ & $\begin{array}{c}\text { OR } \\
(95 \% \text { CoI })\end{array}$ & $\begin{array}{l}x^{2} \\
(p)\end{array}$ & $\begin{array}{c}\text { OR } \\
(95 \% \mathrm{CoI})\end{array}$ \\
\hline$\underset{\leq 26}{\text { MMSE }}$ & $\begin{array}{c}0.5 \\
(0.5)\end{array}$ & $\begin{array}{c}1.4 \\
(0.6-3.5)\end{array}$ & $\begin{array}{c}0.5 \\
(0.5)\end{array}$ & $\begin{array}{c}1.4 \\
(0.5-3.5)\end{array}$ & $\begin{array}{c}0.6 \\
(0.4)\end{array}$ & $\begin{array}{c}1.4 \\
(0.6-3.6)\end{array}$ & $\begin{array}{c}5.0 \\
(0.02)\end{array}$ & $\begin{array}{c}3.0 \\
(1.0-9.0)\end{array}$ & $\begin{array}{c}7.4 \\
(0.007)\end{array}$ & $\begin{array}{c}3.9 \\
(1.3-12.0)\end{array}$ & $\begin{array}{c}5.6 \\
(0.02)\end{array}$ & $\begin{array}{c}3.2 \\
(1.1-9.6)\end{array}$ \\
\hline
\end{tabular}

MMSE, Mini-Mental State Examination; OR, odds ratio; CoI, confidence interval; Model 1, adjusted for DGI (Dietary Guideline Index); Model 2, adjusted for ARFS (Australian Recommended Food Score); Model 3, adjusted for Aust-HEI (Australian Health Eating Index). 


\section{Discussion}

This is the first study to investigate the relationships between sour-taste genetics and cognitive impairment. The findings demonstrate that the presence of the KCNJ2-rs236514 A allele, a variant associated with altered mRNA stability and protein expression of the taste receptor [51-53], increases the likelihood of MMSE scores indicative of MCI in the total cohort and men. Diet quality was not a confounding factor in these relationships. However, the absence of association between female KCNJ2-A allele carriers and MCI (MMSE) may be related to their higher diet-quality scores in all indices.

While there are limitations to a cross-sectional study design, as a first step, these findings provide new directions for research that may inform new management strategies for cognition-related diseases. Integrating these findings with previous related studies on the biology and function of the SNP, taste genetics, and sour taste informs the hypothesis that the KCNJ2-A allele may be reducing overall signal transduction [10,19-24]. In the absence of directly comparable research, results are contextualized by related studies on the KCNJ2 gene, rs236514 SNP, and the structural and biological commonalities in taste, the brain, and cognitive impairment.

Three previous studies have demonstrated expression and dysregulation in taste receptors in the brain of AD [54], schizophrenia [55], and Parkinson's [56] patients that is not reflective of neuronal loss alone. Sour taste is altered in cognition-related diseases with detection and recognition of sour decreasing and taste thresholds increasing in dementia and AD $[14,16]$. Our results suggest that the KCNJ2-rs236514 variant allele may have a role in the cognitive impairment that characterizes these conditions, particularly in light of the SNP's demonstrated impact on sour taste [25]. Furthermore, the decreased sensitivity to sour perception in dementia and AD supports the hypothesis that the SNP may be reducing sour-taste transduction.

Sour-taste transduction involves the release of neurotransmitters commonly altered in cognition-related diseases. Sour compounds stimulate Type III taste cells to release 5-hydroxytryptophan (5-HT), gamma-aminobutyric acid (GABA), and norepinephrine (NE) [57]. Lower levels of GABA [58,59], NE [60], and 5-HT [61,62], as well as reduced 5-HT neurotransmission [63], are found in the brain of cognitively impaired individuals. KCNJ2 genes are highly expressed in the same areas of the brain (cerebral cortex, amygdala, thalamus, hippocampus, and basal ganglia) $[64,65]$ that demonstrate changes in cognitive decline [66-69]. If as hypothesized the KCNJ2-A allele is reducing transduction, the release of 5-HT, GABA, and NE from the extra-oral taste receptors in the brain may be impacted. While these studies indirectly link sour taste, neurotransmitters, and cognitive impairment, direct research in this area has not been undertaken. The possible extra-oral functions of the gene and SNP require further investigation.

Men carrying the KCNJ2-A allele were more likely to meet the MMSE threshold scores for MCI than those not carrying the allele. The singular study available on sour taste and the KCNJ2-rs236514 SNP did not analyze data by sex [10], and neither did the studies demonstrating decreased sensitivity to sour perception in cognition-related diseases [14,16]. However, adjustments for sex were made in all studies, and no significant effects were found $[10,14,16]$. Sex dimorphisms in sour-taste perception have been demonstrated previously in two research projects. Women found sour to be more intense $[49,50]$ and had a higher preference for sourness [50]. The reasons for sex-based differences in sour taste, and in the relationship between the KCNJ2-rs236514 SNP and MCI in our study, are unknown and require further investigation.

In addition to the cross-sectional design of the study, further limitations should be considered. The absence of research for direct comparative analysis means that the findings are contextualized by associated studies and hypotheses. Both genetics and cognitionrelated diseases are multi-factorial. However, the availability of data on relevant potential confounders in this study allowed for adjustments for common associated factors to be made. In addition to the cohort being well-characterized, the sample size was large, the sex distribution was even, and $81.3 \%$ of participants carried the KCNJ2-A allele. Even 
though the results may not be generalizable to wider age groups, the age of participants ( $\geq 65$ years) is relevant to the higher and increasing prevalence of cognition-related diseases in the elderly. Furthermore, the significant relationships between carriage of the KCNJ2-A allele and MCI (MMSE) existed independently of adjustments for age, broadening the scope for application in other age groups.

\section{Conclusions}

Mild-to-severe cognitive impairment (MMSE) was found to be more likely in the presence of the KCNJ2-rs236514 variant (A) allele in this elderly Australian cohort. In the context of previous knowledge correlating altered sour-taste perception to cognitionrelated diseases, this novel study indicates a more fixed genetic link. While not conclusive, this pilot study on a convenient cross-sectional sample suggests that further research is warranted. Studies on relationships between neurotransmitters common to both sour taste and cognition-related disease, extra-oral functions of sour taste receptors particularly in the brain, the SNP's influence on direction and magnitude of transduction, and the role of diet quality are needed to address the gaps in the body of knowledge.

Author Contributions: Conceptualization, C.F. and E.L.B.; data curation, C.F., A.T., M.L. and E.L.B.; formal analysis, C.F. and E.L.B.; funding acquisition, M.V., M.L. and E.L.B.; investigation, C.F., A.T., M.V., M.L. and E.L.B.; methodology, C.F. and E.L.B.; project administration, M.V., M.L. and E.L.B.; resources, M.V., M.L. and E.L.B.; writing—original draft, C.F. and E.L.B.; writing—review and editing, C.F., A.T., Christopher Scarlett, M.V., M.L., T.B. and E.L.B. All authors have read and agreed to the published version of the manuscript.

Funding: This research was conducted as part of the Retirement Health and Lifestyle Study, with initial and ongoing funding provided by the Australian Research Council (G0188386), Central Coast Local Health District Public Health Unit (G0190658/G1700259), UnitingCare Ageing NSW/ACT (G0189230), Urbis Pty Ltd. (G0189232), Valhalla Village Pty Ltd. (G1000936), and Hunter Valley Research Foundation.

Institutional Review Board Statement: The study was conducted according to the Declaration of Helsinki, and ethics was approved by the Human Research Ethics Committee of the University of Newcastle (Reference No. H-2008-0431).

Informed Consent Statement: Informed consent was obtained from all subjects involved in the RHLS study and for future studies drawing on data collected.

Data Availability Statement: Where ethically appropriate, data can be accessed by contacting the corresponding author.

Acknowledgments: The authors acknowledge the role of Paul Roach in the design of the original study and the roles of Charlotte Martin, Zoe Yates, Katrina King, and Suzanne Niblett in sample collection and data management.

Conflicts of Interest: The authors declare no conflict of interest.

\section{References}

1. National Centre for Social and Economic Modelling. Economic Cost of Dementia in Australia 2016-2056; Institute for Governance and Policy Analysis, University of Canberra: Canberra, Australia, 2017.

2. World Health Organisation. Dementia. Available online: https://www.who.int/news-room/fact-sheets/detail/dementia (accessed on 23 January 2020).

3. Mitchell, A.J.; Kemp, S.; Benito-León, J.; Reuber, M. The influence of cognitive impairment on health-related quality of life in neurological disease. Acta Neuropsychiatr. 2010, 22, 2-13. [CrossRef]

4. Hussenoeder, F.S.; Conrad, I.; Roehr, S.; Fuchs, A.; Pentzek, M.; Bickel, H.; Moesch, E.; Weyerer, S.; Werle, J.; Wiese, B.; et al. Mild cognitive impairment and quality of life in the oldest old: A closer look. Qual. Life Res. 2020, 29, 1675-1683. [CrossRef]

5. Caracciolo, B.; Gatz, M.; Xu, W.; Marengoni, A.; Pedersen, N.L.; Fratiglioni, L. Relationship of subjective cognitive impairment and cognitive impairment no dementia to chronic disease and multimorbidity in a nation-wide twin study. J. Alzheimer's Dis. 2013, 36, 275-284. [CrossRef] [PubMed]

6. Chen, X.; Maguire, B.; Brodaty, H.; O'Leary, F. Dietary patterns and cognitive health in older adults: A systematic review. J. Alzheimer's Dis. 2019, 67, 583-619. [CrossRef] 
7. Jiang, X.; Huang, J.; Song, D.; Deng, R.; Wei, J.; Zhang, Z. Increased consumption of fruit and vegetables is related to a reduced risk of cognitive impairment and dementia: Meta-analysis. Front. Aging Neurosci. 2017, 9, 18. [CrossRef]

8. Solfrizzi, V.; Custodero, C.; Lozupone, M.; Imbimbo, B.P.; Valiani, V.; Agosti, P.; Schilardi, A.; D’Introno, A.; La Montagna, M.; Calvani, M.; et al. Relationships of dietary patterns, foods, and micro- and macronutrients with Alzheimer's disease and late-life cognitive disorders: A systematic review. J. Alzheimer's Dis. 2017, 59, 815-849. [CrossRef]

9. Van den Brink, A.C.; Brouwer-Brolsma, E.M.; Berendsen, A.A.M.; Van Den Rest, O. The mediterranean, dietary approaches to stop hypertension (DASH), and mediterranean-DASH intervention for neurodegenerative delay (MIND) diets are associated with less cognitive decline and a lower risk of Alzheimer's disease-A review. Adv. Nutr. 2019, 10, 1040-1065. [CrossRef]

10. Chamoun, E.; Carroll, N.A.; Duizer, L.M.; Qi, W.; Feng, Z.; Darlington, G.; Duncan, A.M.; Haines, J.; Ma, D. The relationship between single nucleotide polymorphisms in taste receptor genes, taste function and dietary intake in preschool-aged children and adults in the Guelph family health study. Nutrients 2018, 10, 990. [CrossRef]

11. Shen, Y.; Kennedy, O.B.; Methven, L. Exploring the effects of genotypical and phenotypical variations in bitter taste sensitivity on perception, liking and intake of brassica vegetables in the UK. Food Qual. Prefer. 2016, 50, 71-81. [CrossRef]

12. Taruno, A.; Nomura, K.; Kusakizako, T.; Ma, Z.; Nureki, O.; Foskett, J.K. Taste transduction and channel synapses in taste buds. Pflügers Archiv. Eur. J. Physiol. 2021, 473, 3-13. [CrossRef]

13. Román, G.; Jackson, R.; Gadhia, R.; Román, A.; Reis, J. Mediterranean diet: The role of long-chain $\omega-3$ fatty acids in fish; polyphenols in fruits, vegetables, cereals, coffee, tea, cacao and wine; probiotics and vitamins in prevention of stroke, age-related cognitive decline, and Alzheimer disease. Rev. Neurol. 2019, 175, 724-741. [CrossRef]

14. Sakai, M.; Kazui, H.; Shigenobu, K.; Komori, K.; Ikeda, M.; Nishikawa, T. Gustatory dysfunction as an early symptom of se-mantic dementia. Dement. Geriatr. Cognit. Dis. Extra 2017, 7, 395-405. [CrossRef]

15. Kouzuki, M.; Ichikawa, J.; Shirasagi, D.; Katsube, F.; Kobashi, Y.; Matsumoto, H.; Chao, H.; Yoshida, S.; Urakami, K. Detection and recognition thresholds for five basic tastes in patients with mild cognitive impairment and Alzheimer's disease dementia. BMC Neurol. 2020, 20, 1-11. [CrossRef]

16. Sakai, M.; Ikeda, M.; Kazui, H.; Shigenobu, K.; Nishikawa, T. Decline of gustatory sensitivity with the progression of Alzheimer's disease. Int. Psychogeriatr. 2016, 28, 511-517. [CrossRef]

17. Ogawa, T.; Irikawa, N.; Yanagisawa, D.; Shiino, A.; Tooyama, I.; Shimizu, T. Taste detection and recognition thresholds in Japanese patients with Alzheimer-type dementia. Auris Nasus Larynx 2017, 44, 168-173. [CrossRef]

18. Lang, C.; Leuschner, T.; Ulrich, K.; Stößel, C.; Heckmann, J.; Hummel, T. Taste in dementing diseases and Parkinsonism. J. Neurol. Sci. 2006, 248, 177-184. [CrossRef]

19. Wise, P.M.; Hansen, J.L.; Reed, D.R.; Breslin, P.A.S. Twin study of the heritability of recognition thresholds for sour and salty taste. Chem. Senses 2007, 32, 749-754. [CrossRef] [PubMed]

20. Törnwall, O.; Silventoinen, K.; Keskitalo-Vuokko, K.; Perola, M.; Kaprio, J.; Tuorila, H. Genetic contribution to sour taste preference. Appetite 2012, 58, 687-694. [CrossRef] [PubMed]

21. Beckett, E.L.; Martin, C.; Yates, Z.; Veysey, M.; Duesing, K.; Lucock, M. Bitter taste genetics—the relationship to tasting, liking, consumption and health. Food Funct. 2014, 5, 3040-3054. [CrossRef] [PubMed]

22. Diószegi, J.; Llanaj, E.; Ádány, R. Genetic background of taste perception, taste preferences, and its nutritional implications: A systematic review. Front. Genet. 2019, 10. [CrossRef]

23. Hibino, H.; Inanobe, A.; Furutani, K.; Murakami, S.; Findlay, I.; Kurachi, Y. Inwardly rectifying potassium channels: Their structure, function, and physiological roles. Physiol. Rev. 2010, 90, 291-366. [CrossRef]

24. Ye, W.; Chang, R.B.; Bushman, J.D.; Tu, Y.-H.; Mulhall, E.M.; Wilson, C.E.; Cooper, A.J.; Chick, W.S.; Hill-Eubanks, D.C.; Nelson, M.T.; et al. The K+ channel KIR2.1 functions in tandem with proton influx to mediate sour taste transduction. Proc. Natl. Acad. Sci. USA 2016, 113, E229-E238. [CrossRef]

25. Chamoun, E.; Mutch, D.M.; Allen-Vercoe, E.; Buchholz, A.C.; Duncan, A.M.; Spriet, L.L.; Haines, J.; Ma, D.W.L. A review of the associations between single nucleotide polymorphisms in taste receptors, eating behaviors, and health. Crit. Rev. Food Sci. Nutr. 2018, 58, 194-207. [CrossRef]

26. Ferraris, C.; Turner, A.; Kaur, K.; Piper, J.; Veysey, M.; Lucock, M.; Beckett, E.L. Salt taste genotype, dietary habits and biomarkers of health: No associations in an elderly cohort. Nutrients 2020, 12, 1056. [CrossRef]

27. Abbott, K.A.; Veysey, M.; Lucock, M.; Niblett, S.; King, K.; Burrows, T.; Garg, M.L. Sex-dependent association between erythrocyte n-3 PUFA and type 2 diabetes in older overweight people. Br. J. Nutr. 2016, 115, 1379-1386. [CrossRef]

28. Beckett, E.L.; Duesing, K.; Martin, C.; Jones, P.; Furst, J.; King, K.; Niblett, S.; Yates, Z.; Veysey, M.; Lucock, M. Relationship between methylation status of vitamin D-related genes, vitamin D levels, and methyl-donor biochemistry. J. Nutr. Intermed. Metab. 2016, 6, 8-15. [CrossRef]

29. Marfell-Jones, M.; Norton, K.; Carter, L.; Olds, T. International Standards for Anthropometric Assessment; International Society for the Advancement of Kinanthropometry: Adelaide, Australia, 2001.

30. Beckett, E.L.; Martin, C.; Boyd, L.; Porter, T.; King, K.; Niblett, S.; Yates, Z.; Veysey, M.; Lucock, M. Reduced plasma homocysteine levels in elderly Australians following mandatory folic acid fortification-A comparison of two cross-sectional cohorts. J. Nutr. Intermed. Metab. 2017, 8, 14-20. [CrossRef]

31. QIAGEN. QIAamp®DNA Mini and Blood Mini Handbook, 3rd ed.; QIAGEN: Hilden, Germany, 2012. 
32. Baek, M.J.; Kim, K.; Park, Y.H.; Kim, S. The validity and reliability of the mini-mental state examination-2 for detecting mild cognitive impairment and Alzheimer's disease in a Korean population. PLoS ONE 2016, 11, e0163792. [CrossRef]

33. Bossers, W.J.; Van Der Woude, L.H.; Boersma, F.; Scherder, E.J.; Van Heuvelen, M.J. Recommended measures for the assessment of cognitive and physical performance in older patients with dementia: A systematic review. Dement. Geriatr. Cogn. Disord. Extra 2012, 2, 589-609. [CrossRef]

34. Arevalo-Rodriguez, I.; Smailagic, N.; Roqué I Figuls, M.; Ciapponi, A.; Sanchez-Perez, E.; Giannakou, A.; Pedraza, O.L.; Bonfill Cosp, X.; Cullum, S. Mini-Mental State Examination (MMSE) for the detection of Alzheimer's disease and other dementias in people with mild cognitive impairment (MCI). Cochrane Database Syst. Rev. 2015, CD010783. [CrossRef]

35. Buchhave, P.; Stomrud, E.; Warkentin, S.; Blennow, K.; Minthon, L.; Hansson, O. Cube copying test in combination with rCBF or CSF A[beta]42 predicts development of Alzheimer's disease. Dement. Geriat. Cognit. Dis. 2008, 25, 544-552. [CrossRef]

36. O'Caoimh, R.; Gao, Y.; Svendovski, A.; Gallagher, P.; Eustace, J.; Molloy, D.W. Comparing approaches to optimize cut-off scores for short cognitive screening instruments in mild cognitive impairment and dementia. J. Alzheimer's Dis. 2017, 57, 123-133. [CrossRef]

37. Palmqvist, S.; Hertze, J.; Minthon, L.; Wattmo, C.; Zetterberg, H.; Blennow, K.; Londos, E.; Hansson, O. Comparison of brief cognitive tests and CSF biomarkers in predicting Alzheimer's disease in mild cognitive impairment: Six-year follow-up study. PLOS ONE 2012, 7, e38639. [CrossRef]

38. Pozueta, A.; Rodríguez-Rodríguez, E.; Vazquez-Higuera, J.L.; Mateo, I.; Sánchez-Juan, P.; González-Perez, S.; Berciano, J.; Combarros, O. Detection of early Alzheimer's disease in MCI patients by the combination of MMSE and an episodic memory test. BMC Neurol. 2011, 11, 78. [CrossRef]

39. Xu, G.; Meyer, J.S.; Thornby, J.; Chowdhury, M.; Quach, M. Screening for mild cognitive impairment (MCI) utilizing combined mini-mental-cognitive capacity examinations for identifying dementia prodromes. Int. J. Geriatr. Psychiatry 2002, 17, 1027-1033. [CrossRef] [PubMed]

40. Dufficy, L.; Naumovski, N.; Ng, X.; Blades, B.; Yates, Z.; Travers, C.; Lewis, P.; Sturm, J.; Veysey, M.; Roach, P.D.; et al. G80A reduced folate carrier SNP influences the absorption and cellular translocation of dietary folate and its association with blood pressure in an elderly population. Life Sci. 2006, 79, 957-966. [CrossRef] [PubMed]

41. Xyris Software. Foodworks. Available online: https:/ / xyris.com.au (accessed on 13 January 2021).

42. National Health \& Medical Research Council. The Australian Dietary Guidelines. Available online: https://www.eatforhealth. gov.au/guidelines (accessed on 13 January 2021).

43. National Health and Medical Research Council. Australian Guide to Healthy Eating. Available online: https://www.eatforhealth. gov.au/guidelines/australian-guide-healthy-eating (accessed on 13 January 2021).

44. Australian Institute of Health and Welfare. Towards National Indicators for Food and Nutrition: An AIHW View-Reporting Against the Dietary Guidelines for Australian Adults. Available online: https:/ /www.aihw.gov.au/reports/food-nutrition/ towards-national-indicators-food-nutrition/contents/table-of-contents (accessed on 13 January 2021).

45. McNaughton, S.A.; Ball, K.; Crawford, D.; Mishra, G.D. An index of diet and eating patterns is a valid measure of diet quality in an Australian population. J. Nutr. 2008, 138, 86-93. [CrossRef] [PubMed]

46. Collins, C.E.; Burrows, T.L.; Rollo, M.E.; Boggess, M.M.; Watson, J.F.; Guest, M.; Duncanson, K.; Pezdirc, K.; Hutchesson, M.J. The comparative validity and reproducibility of a Diet Quality Index for adults: The Australian recommended food score. Nutrents 2015, 7, 785-798. [CrossRef] [PubMed]

47. Australian Institute of Health and Welfare. Australian Diet Quality Index Project; Australian Institute of Health and Welfare: Canberra, Australia, 2007.

48. Puputti, S.; Aisala, H.; Hoppu, U.; Sandell, M. Factors explaining individual differences in taste sensitivity and taste modality recognition among Finnish adults. J. Sens. Stud. 2019, 34. [CrossRef]

49. Fischer, M.E.; Cruickshanks, K.J.; Schubert, C.R.; Pinto, A.; Klein, B.E.K.; Klein, R.; Nieto, F.J.; Pankow, J.S.; Huang, G.-H.; Snyder, D.J. Taste intensity in the Beaver Dam Offspring Study. Laryngoscope 2013, 123, 1399-1404. [CrossRef]

50. Barragán, R.; Coltell, O.; Portolés, O.; Asensio, E.M.; Sorlí, J.V.; Ortega-Azorín, C.; González, J.I.; Sáiz, C.; Fernández-Carrión, R.; Ordovas, J.M.; et al. Bitter, sweet, salty, sour and umami taste perception decreases with age: Sex-specific analysis, modulation by genetic variants and taste-preference associations in 18 to 80 year-old subjects. Nutrients 2018, 10, 1539. [CrossRef]

51. Schwerk, J.; Savan, R. Translating the Untranslated Region. J. Immunol. 2015, 195, 2963-2971. [CrossRef]

52. Steri, M.; Idda, M.L.; Whalen, M.B.; Orrú, V. Genetic variants in mRNA untranslated regions. Wiley Interdiscip. Rev. RNA 2018, 9 , e1474. [CrossRef]

53. National Center for Biotechnology Information National Library of Medicine. Database of Single Nucleotide Polymorphisms (dbSNP). Available online: https:/ / www.ncbi.nlm.nih.gov/snp/ (accessed on 28 January 2021).

54. Ansoleaga, B.; Garcia-Esparcia, P.; Llorens, F.; Moreno, J.; Aso, E.; Ferrer, I. Dysregulation of brain olfactory and taste receptors in AD, PSP and CJD, and AD-related model. Neuroscience 2013, 248, 369-382. [CrossRef]

55. Ansoleaga, B.; Garcia-Esparcia, P.; Pinacho, R.; Haro, J.M.; Ramos, B.; Ferrer, I. Decrease in olfactory and taste receptor expression in the dorsolateral prefrontal cortex in chronic schizophrenia. J. Psychiatr. Res. 2015, 60, 109-116. [CrossRef]

56. Garcia-Esparcia, P.; Schlüter, A.; Carmona, M.; Moreno, J.; Ansoleaga, B.; Torrejón-Escribano, B.; Gustincich, S.; Pujol, A.; Ferrer, I. Functional genomics reveals dysregulation of cortical olfactory receptors in parkinson disease: Novel putative chemoreceptors in the human brain. J. Neuropathol. Exp. Neurol. 2013, 72, 524-539. [CrossRef] 
57. Huang, Y.A.; Pereira, E.; Roper, S.D. Acid stimulation (sour taste) elicits GABA and serotonin release from mouse taste cells. PLoS ONE 2011, 6, e25471. [CrossRef]

58. Porges, E.C.; Woods, A.J.; Edden, R.A.; Puts, N.A.; Harris, A.D.; Chen, H.; Garcia, A.M.; Seider, T.R.; Lamb, D.G.; Williamson, J.B.; et al. Frontal gamma-aminobutyric acid concentrations are associated with cognitive performance in older adults. Biol. Psychiatry: Cogn. Neurosci. Neuroimaging 2017, 2, 38-44. [CrossRef]

59. Huang, D.; Liu, D.; Yin, J.; Qian, T.; Shrestha, S.; Ni, H. Glutamate-glutamine and GABA in brain of normal aged and patients with cognitive impairment. Eur. Radiol. 2016, 27, 2698-2705. [CrossRef]

60. Borodovitsyna, O.; Flamini, M.; Chandler, D. Noradrenergic modulation of cognition in health and disease. Neural Plast. 2017, 2017, 1-14. [CrossRef] [PubMed]

61. Cowen, P.; Sherwood, A.C. The role of serotonin in cognitive function: Evidence from recent studies and implications for understanding depression. J. Psychopharmacol. 2013, 27, 575-583. [CrossRef] [PubMed]

62. Mendelsohn, D.; Riedel, W.J.; Sambeth, A. Effects of acute tryptophan depletion on memory, attention and executive functions: A systematic review. Neurosci. Biobehav. Rev. 2009, 33, 926-952. [CrossRef] [PubMed]

63. Švob Štrac, D.; Pivac, N.; Mück-Šeler, D. The serotonergic system and cognitive function. Transl. Neurosci. 2016, 7, 35-49. [CrossRef] [PubMed]

64. GTExPortal. Gene Expression for KCNJ2. Available online: https://gtexportal.org/home/gene/KCNJ2 (accessed on 24 January 2021).

65. Uhlén, M.; Fagerberg, L.; Hallström, B.M.; Lindskog, C.; Oksvold, P.; Mardinoglu, A.; Sivertsson, Å.; Kampf, C.; Sjöstedt, E.; Asplund, A.; et al. Proteomics. Tissue-based map of the human proteome. Science 2015, 347, 1260419. [CrossRef] [PubMed]

66. Shi, F.; Liu, B.; Zhou, Y.; Yu, C.; Jiang, T. Hippocampal volume and asymmetry in mild cognitive impairment and Alzheimer's disease: Meta-analyses of MRI studies. Hippocampus 2009, 19, 1055-1064. [CrossRef] [PubMed]

67. Poulin, S.P.; Dautoff, R.; Morris, J.C.; Barrett, L.F.; Dickerson, B.C. Amygdala atrophy is prominent in early Alzheimer's disease and relates to symptom severity. Psychiatry Res. Neuroimaging 2011, 194, 7-13. [CrossRef]

68. Ledig, C.; Schuh, A.; Guerrero, R.; Heckemann, R.A.; Rueckert, D. Structural brain imaging in Alzheimer's disease and mild cognitive impairment: Biomarker analysis and shared morphometry database. Sci. Rep. 2018, 8, 1-16. [CrossRef]

69. Leisman, G.; Braun-Benjamin, O.; Melillo, R. Cognitive-motor interactions of the basal ganglia in development. Front. Syst. Neurosci. 2014, 8, 16. [CrossRef] [PubMed] 